\title{
Prophet Inequalities for Averages of Independent Non-Negative Random Variables
}

Theodore P. Hill

School of Mathematics, Georgia Institute of Technology, Atlanta, Georgia 30332, USA

\section{$\S 1$. Introduction}

The main purpose of this paper is to prove the following theorem, which sharpens results of Krengel and Sucheston $[11,12]$ in which the weaker constant $2(1+\sqrt{3})$ was obtained. (Here $E X$ is the expected value of the random variable $X$, and $T_{n}$ and $T$ are the sets of stop rules $\leqq n$, and of a.s. finite stop rules, respectively.)

Theorem 1.1. If $X_{1}, \ldots, X_{n}$ are independent non-negative random variables, then

$$
E\left(\max _{j \leqq n}\left\{\frac{X_{1}+\ldots+X_{j}}{j}\right\}\right) \leqq\left(2-n^{-1}\right) \sup _{t \in T_{n}}\left\{E\left(\frac{X_{1}+\ldots+X_{t}}{t}\right)\right\},
$$

and this bound is sharp for all $n \geqq 1$.

By passing to limits, one easily obtains the following corollary.

Corollary 1.2. If $X_{1}, X_{2}, \ldots$ are independent non-negative random variables, then

$$
E\left(\sup _{n}\left\{\frac{X_{1}+\ldots+X_{n}}{n}\right\}\right) \leqq 2 \sup _{t \in T}\left\{E\left(\frac{X_{1}+\ldots+X_{t}}{t}\right)\right\},
$$

and this constant " 2 " is sharp.

Inequalities such as (1) and (2), which compare the expected supremum of a sequence of random variables with the supremum over stop rules of the expected value at the time of stopping, were first discovered by Krengel and Sucheston $[11,12]$ and have been called "prophet inequalities" because of the probabilistic interpretation of $E\left(\sup Z_{n}\right)$ as the expected value of the sequence $\left\{Z_{n}\right\}$ to a "prophet", or player with complete foresight. Such inequalities have been studied for various processes $\left\{Z_{n}\right\}$ including: $\left\{Z_{n}\right\}$ independent and non- 
negative $[4,5,10,11,12,13] ;\left\{Z_{n}\right\}$ independent and uniformly bounded $[4,6]$; $\left\{Z_{n}\right\}$ i.i.d. $[7,9,13] ;\left\{Z_{n}\right\}$ uniformly bounded martingales and arbitrarily dependent $[2,8]$; and exchangeable random variables $[3]$.

\section{§2. Preliminaries}

Throughout this paper, $X_{1}, X_{2}, \ldots$ are independent non-negative random variables on a probability space $(\Omega, \mathscr{F}, P), E X$ is the expected value of $X, X^{+}$is $\max \{X, 0\}, a \wedge b$ is $\min \{a, b\}$, and $V, M$, and $R$ are the following functionals:

$$
\begin{aligned}
V\left(Z_{1}, \ldots, Z_{n}\right) & =\sup \left\{E\left(Z_{t}\right): t \in T_{n}\right\} ; \\
M\left(Z_{1}, \ldots, Z_{n}\right) & =E\left(\max \left\{Z_{i}: i \leqq n\right\}\right) ; \text { and } \\
R\left(Z_{1}, \ldots, Z_{n}\right) & =M\left(Z_{1}, \ldots, Z_{n}\right) / V\left(Z_{1}, \ldots, Z_{n}\right) .
\end{aligned}
$$

The following result from classical optimal stopping theory (essentially the principle of backward induction) will be used repeatedly, and is included here for ease of reference. (For definitions and a reference see Chap. 3 and 4 of [1].)

Lemma 2.1. Let $Z_{1}, Z_{2}, \ldots$ be integrable random variables. Then

(i) $V\left(Z_{j}, Z_{j+1}, \ldots, Z_{n} \mid Z_{1}, \ldots, Z_{j}\right) \stackrel{\text { a.s. }}{=} \max \left\{Z_{j}, V\left(Z_{j+1}, \ldots, Z_{n} \mid Z_{1}, \ldots, Z_{j}\right)\right\} \quad$ for all $j=1, \ldots, n$; and

(ii) if $t^{*}$ is the stop rule defined by $t^{*} \leqq n$ and $\left\{t^{*}=j<n\right\} \Leftrightarrow t^{*}>j-1$ and $Z_{j}>V\left(Z_{j+1}, \ldots, Z_{n} \mid Z_{1}, \ldots, Z_{j}\right)$, then $E Z_{t^{*}}=V\left(Z_{1}, \ldots, Z_{n}\right)$.

Lemma 2.2. Let $Z_{j+1}, \ldots, Z_{n}$ be independent non-negative random variables. Then there exists a unique constant $\alpha \geqq 0$ such that for every random variable $Z_{j}$ independent of $Z_{j+1}, \ldots, Z_{n}$, there is a stop rule $t^{*}$ with $\left\{t^{*}=1\right\} \Leftrightarrow\left\{Z_{j}>\alpha\right\}$ which is optimal for the sequence $\frac{Z_{j}}{j}, \ldots, \frac{Z_{j}+\ldots+Z_{n-1}}{n-1}, Z_{n}$.

Proof. The function

$$
\psi(x)=j V\left(\frac{x+Z_{j+1}}{j+1}, \ldots, \frac{x+Z_{j+1}+\ldots+Z_{n-1}}{n-1}, Z_{n}\right)
$$

is continuous and increasing, and $\psi(x+h)-\psi(x) \leqq j(j+1)^{-1} h$ for all $x$ and all $h>0$, so $\psi(x) \leqq \psi(0)+j(j+1)^{-1} x$ for all $x \geqq 0$. Since $\psi(0) \geqq 0$, this implies there is a unique non-negative fixed point $\alpha$ of $\psi$, and $x \leqq \psi(x) \Leftrightarrow x \leqq \alpha$. Since the $Z$,'s are independent, Lemma 2.1 implies it is optimal to stop at time 1 in the sequence $\frac{Z_{j}}{j}, \ldots, \frac{Z_{j}+\ldots+Z_{n-1}}{n-1}, Z_{n}$ if and only if

$$
\frac{Z_{j}(\omega)}{j}>V\left(\frac{Z_{j}(\omega)+Z_{j+1}}{j+1}, \ldots, \frac{Z_{j}(\omega)+\ldots+Z_{n-1}}{n-1}, Z_{n}\right)
$$

that is, if and only if $Z_{j}(\omega)>\alpha$. 
Considerable use will also be made of long shot random variables [5], that is, random variables which take on only the value zero (with high probability) and one other non-negative value (with small probability). Whenever needed, it will be assumed (by enlarging the probability space if necessary) that such random variables, even ones which are independent of other given random variables, always exist.

\section{§3. Proof of Theorem 1.1}

Lemma 3.1. For all random variables $X \geqq 0$, all $c \geqq 0$, and all $b \geqq 1$,

$$
R\left(c, \frac{c+X}{b}\right) \leqq 2-b^{-1} \text {. }
$$

Proof. If $c=0$, then $M\left(c, \frac{c+X}{b}\right)=E\left(\frac{X}{b}\right)=V\left(c, \frac{c+X}{b}\right)$, so $R=1$ and the conclusion holds since $b \geqq 1$.

If $c>0$, it may be assumed (by multiplying by $c^{-1}$ and letting $\hat{X}=X / c$ ) that $c=1$.

Case $1.1 \leqq \frac{1+E X}{h}$. Then

$$
\begin{aligned}
M\left(1, \frac{1+X}{b}\right) & \leqq 1+\frac{E X}{b}=\left(\frac{b+E X}{1+E X}\right)\left(\frac{1+E X}{b}\right) \\
& =\left(1+\frac{b-1}{1+E X}\right) \vee\left(1, \frac{1+X}{b}\right) \leqq\left(2-\frac{1}{b}\right) \vee\left(1, \frac{1+X}{b}\right) .
\end{aligned}
$$

Case 2. $1>\frac{1+E X}{b}$. Similar.

For the remainder of this section it will be assumed that $X_{1}, \ldots, X_{n}$ are fixed non-negative independent random variables, and to establish Theorem 1.1 , it may further be assumed that each $X_{i}$ is a simple random variable taking only strictly positive values.

$$
\text { Let: } \begin{aligned}
m & =M\left(X_{1}, \ldots, \frac{X_{1}+\ldots+X_{n}}{n}\right) \\
v & =V\left(X_{1}, \ldots, \frac{X_{1}+\ldots+X_{n}}{n}\right) ; \text { and } \\
r & =m / v .
\end{aligned}
$$

Lemma 3.2. There exist $j<n$, constants $c_{1}, \ldots, c_{j}>0$, and independent non-negative r.v.'s $\hat{X}_{j+1}, \ldots, \hat{X}_{n}$ satisfying

$$
\begin{gathered}
R\left(c_{1}, \ldots, \frac{c_{1}+\ldots+c_{j}}{j}, \frac{c_{1}+\ldots+c_{j}+\hat{X}_{j+1}}{j+1}, \ldots,\right. \\
\left.\quad \frac{c_{1}+\ldots+c_{j}+\hat{X}_{j+1}+\ldots+\hat{X}_{n}}{n}\right) \geqq r
\end{gathered}
$$


and

$$
\frac{c_{1}+\ldots+c_{j}}{j}=V\left(\frac{c_{1}+\ldots+c_{j}}{j}, \ldots, \frac{c_{1}+\ldots+c_{j}+\hat{X}_{j+1}+\ldots+\hat{X}_{n}}{n}\right) .
$$

Proof. First observe that

$$
\begin{aligned}
\hat{R}\left(p_{1}, \ldots, p_{N}\right)= & \left(\sum_{i=1}^{N} p_{i} m_{i}\right) /\left(\sum_{i=1}^{N} p_{i} v_{i}\right) \quad \text { is monotone in } p_{i} \\
& \text { for each } i=1, \ldots, N-1,
\end{aligned}
$$

(where $\left.p_{N}=1-\sum_{i=1}^{N-1} p_{i}\right)$.

Suppose $X_{1}$ takes values $\left\{a_{i}\right\}_{i=1}^{N}$ with probabilities $\left\{p_{i}\right\}_{i=1}^{N}$ respectively, and apply (5) with

$$
m_{i}=M\left(a_{i}, \frac{a_{i}+X_{2}}{2}, \ldots, \frac{a_{i}+X_{2}+\ldots+X_{n}}{n}\right)
$$

and

$$
v_{i}=V\left(a_{i}, \ldots, \frac{a_{i}+X_{2}+\ldots+X_{n}}{n}\right)
$$

to conclude first that, as a function of $p_{1}, \hat{R}$ is maximized (for probabilities $\left.\left\{p_{i}\right\}_{i=1}^{N}\right)$ when $p_{1}=0$ or $p_{1}=1-\sum_{i=2}^{N-1} p_{i}$. Continuing with $p_{2}, p_{3}, \ldots, p_{N-1}$ one eventually concludes that $\hat{R}$ is maximized when $p_{i}=1$ for some $i$. (Actually the somewhat simpler inequality $\left(\sum_{i=1}^{N} p_{i} m_{i}\right) /\left(\sum_{i=1}^{N} p_{i} v_{i}\right) \leqq \max _{i \leqq N} m_{i} / v_{i}$ can be used to show this, but the monotonicity argument given here will be necessary in later steps.)

Thus it may now be assumed (in order to prove (3) and (4)) that $X_{1}=c_{1}$ a.s. for some constant $c_{1} \in\left\{a_{1}, \ldots, a_{N}\right\}$. If

$$
c_{1}=V\left(c_{1}, \frac{c_{1}+X_{2}}{2}, \ldots, \frac{c_{1}+X_{2}+\ldots+X_{n}}{n}\right)
$$

then (3) and (4) are satisfied with $j=1$ and $\left\{\hat{X}_{i}\right\}=\left\{X_{i}\right\}$.

On the other hand, if $c_{1}<V\left(c_{1}, \ldots, \frac{c_{1}+X_{2}+\ldots+X_{n}}{n}\right)$, then $t^{*} \geqq 2$ a.s. for any optimal stop rule for the sequence $c_{1}, \frac{c_{1}+X_{2}}{2}, \ldots, \frac{c_{1}+X_{2}+\ldots+X_{n}}{n}$, so if $X_{2}$ takes values $\left\{b_{i}\right\}_{i=1}^{N}$ with probabilities $\left\{q_{i}\right\}_{i=1}^{N}$ respectively, then letting

$$
m_{i}=M\left(c_{1}, \frac{c_{1}+b_{i}}{2}, \ldots, \frac{c_{1}+b_{i}+X_{3}+\ldots+X_{n}}{n}\right)
$$

and

$$
v_{i}=V\left(\frac{c_{1}+b_{i}}{2}, \ldots, \frac{c_{1}+b_{i}+X_{3}+\ldots+X_{n}}{2}\right),
$$


it follows that

$$
R\left(c_{1}, \frac{c_{1}+X_{2}}{2}, \ldots, \frac{c_{1}+X_{2}+\ldots+X_{n}}{n}\right)=\left(\sum_{i=1}^{N} q_{i} m_{i}\right) /\left(\sum_{i=1}^{N} q_{i} v_{i}\right) .
$$

Applying (5) again, $R$ may be increased by increasing or decreasing $q_{1}$ (depending on whether $R$ is a monotone increasing or monotone decreasing function of $q_{1}$ ) until either:

$$
c_{1}=V\left(c_{1}, \frac{c_{1}+\hat{X}_{2}}{2}, \ldots, \frac{c_{1}+\hat{X}_{2}+X_{3}+\ldots+X_{n}}{n}\right)
$$

or

$$
q_{1}=0, \quad \text { or } \quad q_{1}=1-\sum_{i=2}^{N-1} q_{i} .
$$

If (6) obtains, then (3) and (4) are satisfied with $j=1, \hat{X}_{2}$ as modified, and $\hat{X}_{j}$ $=X_{j}$ for $j>2$. Otherwise (i.e., if (7) obtains) then increase $R$ by changing $q_{2}$ in the same manner, then $q_{3}$ if necessary, etc. Eventually either (6) holds, or $q_{1}=1$ for some $i$, in which case $\hat{X}_{2}=c_{2}$ a.s. for some constant $c_{2} \in\left\{b_{1}, \ldots, b_{N}\right\}$. Proceeding as before, if

$$
\frac{c_{1}+c_{2}}{2}=V\left(\frac{c_{1}+c_{2}}{2}, \ldots, \frac{c_{1}+c_{2}+X_{3}+\ldots+X_{n}}{n}\right)
$$

then (3) and (4) are satisfied with $j=2$ and $\left\{\hat{X}_{j}\right\}=\left\{X_{j}\right\}$. If

$$
\frac{c_{1}+c_{2}}{2}<V\left(\frac{c_{1}+c_{2}}{2}, \ldots, \frac{c_{1}+c_{2}+X_{3}+\ldots+X_{n}}{n}\right),
$$

continue this procedure with $X_{3}$, then $X_{4}$ if necessary, and so on. Eventually either (4) will hold for some $j<n$, or else there exist constants $c_{1}, \ldots, c_{n-1}>0$ so that (3) holds with $j=n-1$, and

$$
V\left(c_{1}, \ldots, \frac{c_{1}+\ldots+c_{n-1}+\hat{X}_{n}}{n}\right)=E\left(\frac{c_{1}+\ldots+c_{n-1}+\hat{X}_{n}}{n}\right) .
$$

In this last case, letting $\frac{c_{1}+\ldots+c_{k}}{k}=\max _{i<n} \frac{c_{1}+\ldots+c_{i}}{i}$, and $c=c_{1}+\ldots+c_{k}$ and $X=c_{k+1}+\ldots+c_{n-1}+\hat{X}_{n}$, one has that

$$
V\left(c_{1}, \ldots, \frac{c_{1}+\ldots+c_{n-1}+\hat{X}_{n}}{n}\right)=V\left(\frac{c}{k}, \frac{c+X}{n}\right)
$$

and

$$
M\left(c_{1}, \ldots, \frac{c_{1}+\ldots+c_{n-1}+\hat{X}_{n}}{n}\right)=M\left(\frac{c}{k}, \frac{c+X}{n}\right) .
$$


Since $\frac{c}{k}<\frac{c+E X}{n}$ (otherwise (4) held for $j=k$ ), decreasing $X$ to $\hat{X}=\lambda X$ $(0<\lambda<1)$ so that $\frac{c}{k}=\frac{c+E \hat{X}}{n}$ decreases $V$ by exactly $\delta=\frac{(1-\lambda)}{n} E X>0$, and $M$ by at most $\delta$. This results in an increase in $R$, since $(M-\delta) /(V-\delta) \geqq M / V$ for $V \leqq M$ and $\delta>0$ (an argument used in Lemma 1 of [5]). Then (3) and (4) are satisfied with: $c_{i}=0$ for all $i \neq k ; c_{k}=c ; \hat{X}_{n}=\hat{X}$. (An alternative proof of this last step, where $X_{i}=c_{i}$ a.s. for $i \leqq n-1$, can be based on Lemma 3.1.)

Lemma 3.3. There exist a constant $c>0$, integer $k<n$, and random variables $Y_{k+1}, \ldots, Y_{n} \geqq 0$, with $Y_{n}$ independent of $Y_{k+1}, \ldots, Y_{n-1}$ so that

$$
\begin{gathered}
R\left(\frac{c}{k}, \frac{Y_{k+1}}{k+1}, \ldots, \frac{Y_{n-1}}{n-1}, \frac{c+Y_{n}}{n}\right) \geqq r ; \quad \text { and } \\
V\left(\frac{c}{k}, \frac{Y_{k+1}}{k+1}, \ldots, \frac{Y_{n-1}}{n-1}, \frac{c+Y_{n}}{n}\right)=\frac{c}{k}=E\left(\frac{c+Y_{n}}{n}\right) .
\end{gathered}
$$

Proof. Let $j<n, c_{1}, \ldots, c_{j}$, and $\left\{X_{i}\right\}_{j+1}^{n}=\left\{\hat{X}_{i}\right\}_{j+1}^{n}$ be as in Lemma 3.2, and let $k \leqq j$ be such that

$$
\frac{c_{1}+\ldots+c_{k}}{k}=\max _{i \leqq j} \frac{c_{1}+\ldots+c_{i}}{i} .
$$

Then, by increasing $X_{n}$ if necessary (i.e., if $\frac{c_{1}+\ldots+c_{k}}{k}>\frac{c_{1}+\ldots+c_{j}}{j}$ ), it may be assumed that (3) and (4) hold with $j=k$. (The argument if $X_{n}$ is modified is this. If $\alpha, \beta, \gamma_{1}, \gamma_{2}, \ldots$ are constants with $\alpha<\beta$ and $\gamma_{i} \leqq \beta$ then $\alpha=V\left(\alpha, W_{1}, \ldots, W_{n}\right)$ implies there exists a $\delta>0$ with

$$
\begin{aligned}
\beta & =V\left(\alpha, W_{1}, \ldots, W_{n}+\delta\right)=V\left(\beta, \gamma_{1}, \ldots, \gamma_{k}, \alpha, W_{1}, \ldots, W_{n}\right) \\
& =V\left(\beta, \gamma_{1}, \ldots, \gamma_{k}, \alpha, W_{1}, \ldots, W_{n}+\delta\right) .
\end{aligned}
$$

Since adding $\delta$ to $W_{n}$ keeps $V$ the same, and can only increase $M$, this can only result in an increase in $R$.)

Next observe that if $X_{n}$ is replaced by a long shot $L_{k}$ (independent of $\left.X_{1}, \ldots, X_{n-1}\right)$ with $P\left(L_{k}=\frac{E X_{n}}{\varepsilon}\right)=\varepsilon=1-P\left(L_{k}=0\right)$, then setting $c=c_{1}+\ldots+c_{k}$
one has

$$
V\left(\frac{c}{k}, \ldots, \frac{c+X_{k+1}+\ldots+X_{n-1}}{n-1}, \frac{c+L_{k}}{n}\right)=\frac{c}{k}=v \quad \text { for all } \varepsilon
$$

and

$M\left(\frac{c}{k}, \ldots, \frac{c+X_{k+1}+\ldots+X_{n-1}}{n-1}, \frac{c+L_{k}}{n}\right)>m \quad$ for sufficiently small $\varepsilon$

(Conclusion (10) follows easily from (4), from the fact that $V$ depends on $X_{n}$ only through $E X_{n}$ (by Lemma 2.1), and since eliminating the non-negative term 
$X_{k+1}+\ldots+X_{n-1}$ in the last position cannot increase $V ;(11)$ follows since the independence and strict positivity of the $X_{i}$ 's imply

$$
\begin{array}{rl}
\lim _{\varepsilon \rightarrow 0} & M\left(\frac{c}{k}, \ldots, \frac{c+X_{k+1}+\ldots+X_{n-1}}{n-1}, \frac{c+L_{k}}{n}\right)=\frac{E X_{n}}{n} \\
+ & M\left(\frac{c}{k}, \ldots, \frac{c+X_{k+1}+\ldots+X_{n-1}}{n-1}\right) \\
> & \left.M\left(\frac{c}{k}, \ldots, \frac{c+X_{k+1}+\ldots+X_{n}}{n}\right)=m .\right)
\end{array}
$$

If $E\left(\frac{c+L_{k}}{n}\right)=\frac{c}{k},(8)$ and (9) follow from (10) and (11) taking $Y_{n}=L_{k}$ and $Y_{i}$ $=c+X_{k+1}+\ldots+X_{i}$ for $k<i<n$. Otherwise, by increasing $L_{k}$ if necessary, it may be assumed from (10) that

$$
V\left(\frac{c+X_{k+1}}{k+1}, \ldots, \frac{c+X_{k+1}+\ldots+X_{n-1}}{n-1}, \frac{c+L_{k}}{n}\right)=\frac{c}{k}=v
$$

Let $j=k+1, Z_{i}=X_{i}$ for $k+1<i<n$, and $Z_{n}=\left(c+L_{k}\right) / n$; since $Z_{j+1}, \ldots, Z_{n}$ are independent and $\geqq 0$, Lemma 2.2 implies the existence of a unique constant $\alpha \geqq 0$ (the "optimal cutoff value") such that for every random variable $Z_{j}(=c$ $\left.+X_{k+1}\right)$ independent of $Z_{j+1}, \ldots, Z_{n}$ there is a stop rule $t^{*}$ with $\left\{t^{*}=1\right\} \Leftrightarrow\{c$ $\left.+X_{k+1}>\alpha\right\}$ which is optimal for the sequence $\frac{Z_{j}}{j}, \ldots, \frac{Z_{j}+\ldots+Z_{n-1}}{n-1}, Z_{n}$, that is, for $\frac{c+X_{k+1}}{k+1}, \ldots, \frac{c+X_{k+1}+\ldots+X_{n-1}}{n-1}, \frac{c+L_{k}}{n}$.

Letting $Y_{k+1}=\left(c+X_{k+1}\right) \wedge \alpha$, then

$$
\begin{aligned}
v- & V\left(\frac{Y_{k+1}}{k+1}, \ldots, \frac{Y_{k+1}+X_{k+2}+\ldots+X_{n-1}}{n-1}, \frac{c+L_{k}}{n}\right) \\
= & E \frac{\left(c+X_{k+1}-\alpha\right)^{+}}{k+1} \\
\geqq & M\left(\frac{c}{k}, \ldots, \frac{c+X_{k+1}+\ldots+X_{n-1}}{n-1}, \frac{c+L_{k}}{n}\right) \\
& -M\left(\frac{c}{k}, \frac{Y_{k+1}}{k+1}, \ldots, \frac{Y_{k+1}+X_{k+2}+\ldots+X_{n-1}}{n-1}, \frac{c+L_{k}}{n}\right) \\
& >m-M\left(\frac{c}{k}, \frac{Y_{k+1}}{k+1}, \ldots, \frac{Y_{k+1}+X_{k+2}+\ldots+X_{n-1}}{n-1}, \frac{c+L_{k}}{n}\right),
\end{aligned}
$$

where the strict inequality follows from (11).

Since $V\left(W_{1}, \ldots, W_{n-1}, W_{n}+\delta\right) \leqq V\left(W_{1}, \ldots, W_{n}\right)+\delta$ for all $\left\{W_{i}\right\}$ and $\delta$, this implies the existence of a constant $\gamma \geqq \frac{n}{k+1} E\left(c+X_{k+1}-\alpha\right)^{+}$and a long shot 
$L_{k+1}$ with $E L_{k+1}=\gamma$ (and independent of $L_{k}, Y_{k+1}, X_{k+2}, \ldots, X_{n-1}$ ) satisfying

$$
\begin{aligned}
& V\left(\frac{Y_{k+1}}{k+1}, \ldots, \frac{Y_{k+1}+X_{k+2}+\ldots+X_{n-1}}{n-1}, \frac{c+L_{k}+\gamma}{n}\right) \\
& \quad=V\left(\frac{Y_{k+1}}{k+1}, \ldots, \frac{Y_{k+1}+X_{k+2}+\ldots+X_{n-1}}{n-1}, \frac{c+L_{k}+L_{k+1}}{n}\right)=\frac{c}{k}=v ;
\end{aligned}
$$

and

$$
M\left(\frac{c}{k}, \frac{Y_{k+1}}{k+1}, \ldots, \frac{Y_{k+1}+X_{k+2}+\ldots+X_{n-1}}{n-1}, \frac{c+L_{k}+L_{k+1}}{n}\right)>m .
$$

By the definition of $\alpha$ and $Y_{k+1}$, and the fact that the addition of $L_{k+1}$ only increases the value after time 1 in the sequence in (14), it is seen that

$$
\begin{aligned}
V & \left(\frac{c}{k}, \frac{Y_{k+1}}{k+1}, \ldots, \frac{Y_{k+1}+X_{k+2}+\ldots+X_{n-1}}{n-1}, \frac{c+L_{k}+L_{k+1}}{n}\right) \\
& =V\left(\frac{Y_{k+1}}{k+1}, \ldots, \frac{Y_{k+1}+X_{k+2}+\ldots+X_{n-1}}{n-1}, \frac{c+L_{k}+L_{k+1}}{n}\right) \\
& =V\left(\frac{Y_{k+1}+X_{k+2}}{k+2}, \ldots, \frac{Y_{k+1}+X_{k+2}+\ldots+X_{n-1}}{n-1}, \frac{c+L_{k}+L_{k+1}}{n}\right)=\frac{c}{k}=v .
\end{aligned}
$$

If $E\left(\frac{c+L_{k}+L_{k+1}}{n}\right)=\frac{c}{k},(8)$ and (9) follow from (15) and (16) taking $Y_{n}=L_{k}$ $+L_{k+1}$ and $Y_{i}=\frac{Y_{k+1}+X_{k+2}+\ldots+X_{i}}{i}$ for $k+1<i<n$. Otherwise continue in this manner (i.e., let $\beta$ be the optimal cutoff at time 1 for the third sequence in (16), let $Y_{k+2}=\left(Y_{k+1}+X_{k+2}\right) \wedge \beta$, and let $L_{k+2}$ be a long shot with $E L_{k+2} \geqq \frac{n}{k+2} E\left(Y_{k+1}+X_{k+2}-\beta\right)^{+}$, etc.) to eventually arrive at a $j, k+1<j<n$, (possibly dependent) random variables $Y_{k+2}, \ldots, Y_{j}$ and long shots $L_{k+2}, \ldots, L_{j}$ independent of each other and the $Y_{i}^{\prime}$ s, which satisfy both

$$
\begin{aligned}
V\left(\frac{c}{k}\right. & \left.\frac{Y_{k+1}}{k+1}, \ldots, \frac{Y_{j}}{j}, \frac{Y_{j}+X_{j+1}}{j+1}, \ldots, \frac{Y_{j}+X_{j+1}+\ldots+X_{n-1}}{n-1}, \frac{c+L_{k}+\ldots+L_{j}}{n}\right) \\
\quad & =V\left(\frac{Y_{k+1}}{k+1}, \ldots, \frac{c+L_{k}+\ldots+L_{j}}{n}\right) \\
& =V\left(\frac{Y_{j}}{j}, \ldots, \frac{c+L_{k}+\ldots+L_{j}}{n}\right)=E\left(\frac{c+L_{k}+\ldots+L_{j}}{n}\right)=\frac{c}{k}=v
\end{aligned}
$$

and

$$
M\left(\frac{c}{k}, \frac{Y_{k+1}}{k+1}, \ldots, \frac{Y_{j}}{j}, \frac{Y_{j}+X_{j+1}}{j+1}, \ldots, \frac{Y_{j}+X_{j+1}+\ldots+X_{n-1}}{n-1}, \frac{c+L_{k}+\ldots+L_{j}}{n}\right)>m .
$$


Taking $Y_{i}=Y_{j}+X_{j+1}+\ldots+X_{i}$ for $i=j+1, \ldots, n-1$ and $Y_{n}=L_{k}+\ldots+L_{j}$ completes the proof of (8) and (9) since $r=m / v$. (The terms $\frac{c}{k}, \frac{Y_{k+1}}{k+1}, \ldots, \frac{Y_{j}}{j}$ may be kept in $V$ since it is optimal never to stop at times $k+1, \ldots, j$, as each $Y_{i}$ is $\leqq$ optimal cutoff, and increasing the last random variable preserves this property - this is the same argument as for (16).)

Proof of Theorem 1.1. Fix $n>1$, and recall that the (independent non-negative) random variables $X_{1}, \ldots, X_{n}$ may be assumed to take only a finite number of strictly positive values.

By Lemma 3.3, there exist a constant $c>0$, an integer $k<n$, and nonnegative random variables $Y_{k+1}, \ldots, Y_{n}$, with $Y_{n}$ independent of $Y_{k+1}, \ldots, Y_{n-1}$, which satisfy (8) and (9).

By (9), $\frac{Y_{i}}{i} \leqq \frac{c}{k}$ a.s. for all $i=k+1, \ldots, n-1$ (for otherwise, since $E\left(\frac{c+Y_{n}}{n}\right)=\frac{c}{k}$, it would follow that $V>\frac{c}{k}$ ), so

$$
\begin{gathered}
V\left(\frac{c}{k}, \frac{Y_{k+1}}{k+1}, \ldots, \frac{Y_{n-1}}{n-1} \frac{c+Y_{n}}{n}\right)=V\left(\frac{c}{k}, \frac{c+Y_{n}}{n}\right) ; \quad \text { and } \\
M\left(\frac{c}{k}, \frac{Y_{k+1}}{k+1}, \ldots, \frac{Y_{n-1}}{n-1}, \frac{c+Y_{n}}{n}\right)=M\left(\frac{c}{k}, \frac{c+Y_{n}}{n}\right) .
\end{gathered}
$$

Then by (8), (19), and (20), Lemma 3.1 (first multiplying by $k$, and then setting $b=n / k$ and $\left.X=Y_{n}\right)$ implies $r \leqq 2-n^{-1} k$, so the conclusion $M \leqq(2$ $-n^{-1}$ ) $V$ follows from the definition of $r$ and the fact that $k \geqq 1$.

To see this inequality is sharp, let $X_{1}=1$ a.s., $X_{2}=X_{3}=\ldots=X_{n-1}=0$ a.s., and let $P\left(X_{n}=\frac{n-1}{\varepsilon}\right)=\varepsilon=1-P\left(X_{n}=0\right)$. Then $V\left(X_{1}, \ldots, \frac{X_{1}+\ldots+X_{n}}{n}\right)=1$ and $M\left(X_{1}, \ldots, \frac{X_{1}+\ldots+X_{n}}{n}\right)=2-n^{-1}-\varepsilon+n^{-1} \varepsilon$.

Remarks. The same techniques can be used to prove analogs of (1) and (2) for any sequence of normalizing constants $\left\{a_{n}\right\}$, and that " 2 " is always the best possible bound in (2) if $a_{n} \rightarrow \infty$. An easy modification of Example 1 of [5] shows that without the assumption of non-negativity, no finite bounds for (1) and (2) are possible.

Acknowledgements. The author is grateful to Professors Robert Kertz and Ulrich Krengel for a number of suggestions, and to the Mathematics Department of the University of Leiden for its hospitality and technical assistance during the academic year 1982-83.

\section{References}

1. Chow, Y., Robbins, H., Siegmund, D.: Great expectations: the theory of optimal stopping. Boston, Mass.: Houghton Mifflin, 1971 
2. Cox, D., Kertz, R.: Prophet regions and sharp inequalities for $p$-th absolute moments of martingales. To appear in J. Multivariate Anal.

3. Elton, J., Kertz, R.: Comparison of stop rule and maximum expectations for finite sequences of exchangeable random variables, preprint (1985)

4. Hill, T.: Prophet inequalities and order selection in optimal stopping problems. Proc. Am. Math. Soc. 88, 131-137 (1983)

5. Hill, T., Kertz, R.: Ratio comparisons of supremum and stop rule expectations. Z. Wahrscheinlichkeitstheor. Verw. Geb. 56, 283-285 (1981)

6. Hill, T., Kertz, R.: Additive comparisons of stop rule and supremum expectations of uniformly bounded independent random variables, Proc. Am. Math. Soc. 83, 582-585 (1981)

7. Hill, T., Kertz, R.: Comparisons of stop rule and supremum expectations of i.i.d. random variables, Ann. Probab. 10, 336-345 (1982)

8. Hill, T., Kertz, R.: Stop rule inequalities for uniformly bounded sequences of random variables. Trans. Am. Math. Soc. 278, 197-207 (1983)

9. Kertz, R.: Stop rule and supremum expectations of i.i.d. random variables: a complete comparison by conjugate duality, to appear in J. Multivariate Anal.

10. Kennedy, D.: Optimal stopping of independent random variables and maximizing prophets. Ann. Probab. 13, 566-571 (1985)

11. Krengel, U., Sucheston, L.: Semiamarts and finite values. Bull. Am. Math. Soc. 83, 745-747 (1977)

12. Krengel, U., Sucheston, L.: On semiamarts, amarts, and processes with finite value. Adv. in Probab. Related Topics 4, 197-266 (1978)

13. Samuel-Cahn, E.: Comparison of threshold stop rules and maximum for independent nonnegative random variables. Ann. Probab. 12, 1213-1216 (1984) 